\title{
Study of PTCH1 Gene as a Prognostic Marker to Predict Imatinib Response in CML Patients: a Single Egyptian Center Experience Mohamed Mahmoud Moussa ${ }^{1}$, Mohamed Tarif Hamza², Omnia Shoukry Abdel Fattah ${ }^{1}$, Mary Gamal Naguib*1 \\ Departments of ${ }^{1}$ Internal Medicine-Clinical Hematology and ${ }^{2}$ Clinical Pathology, Faculty of Medicine, Ain Shams University, Egypt \\ *Corresponding author: Mary Gamal, Mobile: (+20) 01224440277, E-mail: m.s.wilson.mg@gmail.com
}

\begin{abstract}
Background: Chronic myeloid leukemia (CML) is a myeloproliferative illness marked by a reciprocal translocation between chromosomes 9 and 22 [t (9;22) (q34; q11)], forming the Philadelphia chromosome and bringing together the Breakpoint Cluster Region (BCR) and Abelson (ABL1) genes. The protein expressed by this fusion gene is a dysregulated tyrosine kinase that causes alterations in cell proliferation, DNA repair, differentiation, and cell cycle by phosphorylating many downstream proteins. Objectives: To assess PTCH1 gene expression and response of adult patients with chronic myeloid leukemia to imatinib treatment.
\end{abstract}

Patients and Methods: This is a prospective case-control study that included 55 subjects; 45 patients with chronic phase chronic myeloid leukemia and 10 healthy age and sex-matched controls. The control group presented from the same geographic origin of the patients in the study group. The study was done between June 2015 and July 2017 at the hematology clinic, Ain Shams University Hospital.

Results: PTCH1 gene was more in cases than control with a statistical significance (P-value=0.024). On following up our cases after 6 months treatment with imatinib, patients with desired response to imatinib treatment had a mean of PTCH1 gene expression of 2.41, compared to 3.58 in patients who did not achieve desired response with a statistical significance with $\mathrm{p}$-value $=0.017$. Incidence of imatinib failure after 6 months of treatment in patients with high PTCH1 expression is $27.8 \%$ while in those with low expression is $44.4 \%$.

Conclusion: The study demonstrates that level of PTCH 1 did not affect imatinib response. Therefore, PTCH1 is not a valid biomarker for imatinib resistance in CML patients.

Keywords: Chronic Phase, Egyptian CML Patients, Predict Imatinib Response, Prognostic Marker, PTCH1 Gene.

\section{INTRODUCTION}

Chronic myeloid leukemia (CML) is a myeloproliferative condition characterised by increased granulocytic cell proliferation without loss of differentiation potential. It is responsible for $20 \%$ of all adult leukemia cases. CML's clinical symptoms are subtle, evolving as the illness develops through its three stages (chronic, accelerated, and blastic). Fatigue, weight loss, low-grade fever, and splenomegaly are signs and symptoms of the chronic phase ${ }^{(\mathbf{1})}$.

CML was the first human malignancy associated with a specific genetic lesion, the Philadelphia chromosome, which harbors the BCR$\mathrm{ABL}$ oncogene. In the realm of hematologic neoplasias, it has now become a paradigm for finding molecular pathways and designing targeted therapeutic strategies (2).

CML strikes at a median age of 50 years, with the highest incidence occurring between the ages of 50 and 60. There is no preference for one gender over another. The chronic phase of the disease is followed by the blastic phase, which is a second, more acute or sudden phase of the disease. The accelerated phase, which occurs occasionally between the chronic and blast phases and is characterised by a more gradual increase in blast cells in the peripheral blood, progressive anemia, thrombocytopenia, and increasing splenomegaly, is characterised by a more gradual increase in blast cells in the peripheral blood, progressive anemia, thrombocytopenia, and increasing splenomegaly. The median life expectancy is around 3 to 5 years ${ }^{(2)}$.

A complete blood count, which reveals an increase in granulocytes of all sorts, including mature myeloid cells, is frequently used to diagnose CML. The presence of basophils and eosinophils is practically ubiquitous, which may help distinguish CML from a leukemoid response. CML is characterized by genetic aberration resulted in the formation of Philadelphia chromosome, which is formed by the fusion of 2 genes, ABL gene and BCR gene together, they are found on chromosome 9 and chromosome 22 respectively. The BCR-ABL1 fusion gene produces the BCR-ABL1 fusion protein, which is a unique gene product. This protein product has an enzyme domain with tyrosine kinase catalytic activity from normal ABL1. This unregulated tyrosine kinase has been linked to CML pathogenesis ${ }^{(3)}$.

The drugs used to treat CML patients are designed to postpone the start of the accelerated or blastic phase of the disease. To achieve hematologic remission, this has typically included a myelosuppressive medication, but more effective drugs -most recently, interferon alfa and targeted treatment with tyrosine kinase inhibitors like imatinib mesylatehave gained prominence. Chemotherapy is sometimes used to prepare for bone marrow or hematopoietic stem cell transplantation. At 3, 6, 12, and 18 months, 
cytogenetic monitoring is necessary. Molecular monitoring is required every 3 months. The degree and timing of hematological, cytological, and molecular findings were used to classify the response to first-line imatinib as ideal, poor, or failing ${ }^{(4)}$.

PTCH1 is a component of the Hedgehog $(\mathrm{Hh})$ signaling pathway, which is a key regulator of cell proliferation, cell surveillance, embryonic development, adult tissue homeostasis, and stem cell quiescence ${ }^{(5)}$.

The patched homolog1 (PTCH1) gene directs the production of the patched-1 protein, which serves as a receptor. Certain other proteins, known as ligands, fit into particular locations on receptor proteins like keys into locks. The patched-1 receptor is liganded by a protein called Sonic Hedgehog. Ligands and receptors work together to send out signals that alter cell growth and function. The gene PTCH1 is known as a tumour suppressor gene. Its Cytogenetic Location: 9q22.32, which is chromosome 9's long (q) arm at location 22.32 (6). In individuals with CP-CML, PTCH1 gene expression at diagnosis should be regarded as a viable molecular marker for predicting the likelihood of imatinib response.

These findings may make it easier for physicians to start a first-line tyrosine kinase inhibitors (TKI) treatment for a specific patient. The expression of the PTCH1 gene does not appear to be linked to the dasatinib response, and its link to the nilotinib response has not been investigated. If there is no link between PTCH1 expression and nilotinib response, PTCH1 expression might be used to guide first-line TKI treatment; if low PTCH1 expression is observed at diagnosis, a second-generation TKI would be a preferable alternative ${ }^{(5)}$.

The aim of our study was to assess PTCH1 gene expression and its relation to the response of adult patients with chronic myeloid leukemia to imatinib treatment.

\section{PATIENTS AND METHODS}

This is a prospective case-control study that included 55 subjects; 45 patients were newly diagnosed chronic myeloid leukemia (chronic phase) and 10 healthy age and sex-matched controls. The control group presented from the same geographic origin of the patients in the study group.

The study was done between June 2015 and July 2017 at the hematology clinic, Ain Shams University Hospital.

\section{Ethical approval:}

An approval of the study was obtained from Ain Shams University academic and ethical committee. Participants gave informed consent for the withdrawal and use of biological samples.

Following diagnosis of Ph-positive CP-CML, patients were started on imatinib, $400 \mathrm{mg}$ daily. All patients participating in the study were subjected initially to: History taking with special emphasize on constitutional symptoms such as fatigue, anorexia, night fever, night sweating, loss of weight, loss of appetite and left abdominal pain, clinical examination including abdominal and lymph node examination, CBC using an automated analyzer with blood film using Giemsa stain, pelviabdominal ultrasound with special emphasize on liver and spleen size, viral markers including HBsAg, HCVAb and HIVAb, comprehensive biochemistry tests including liver function, kidney function tests and LDH were obtained before the start of therapy, bone marrow aspirate stained by Giemsa stain with assessment of blast percentage to determine the stage of CML either chronic phase $<5 \%$ blasts, accelerated phase $>5 \%$ and $<20 \%$ blasts and blastic phase $\geq 20 \%$ blasts and basophil percentage, cytogenetic studies were performed on bone marrow by FISH technique (Fluorescent in situ hybridization) and karyotyping at the time of diagnosis. PCR for BCR$\mathrm{ABL}$ (by quantitative reverse transcriptase polymerase chain reaction) and PCR for PTCH1 mRNA is done for all patients at the time.

All patients were followed up for about 6 months by CBC monthly, physical examination and PCR for BCR-ABL every 3 months.

\section{Patients Monitoring and Response to Therapy:}

We followed up our patients after starting imatinib therapy by CBC and PCR for BCR-ABL every 3 months, searching for hematological remission after 3 months, which is reached by normalization of CBC picture and searching for desired molecular response at 6 months post-treatment according to NCCN guidelines as follows ${ }^{(7)}$ :

- 3 months: BCR-ABL1 transcripts $\leq 10 \%$ by qPCR.

- 6 months: BCR-ABL1 transcripts $\leq 10 \%$ by qPCR.

- 12 months: $\leq 1 \%$ BCR-ABL1.

- 15 months: $\leq 1 \%$ BCR-ABL1.

\section{Methods:}

PTCH1 mRNA detection: detection of RNA transcript level by real time RT-PCR:

1. RNA extraction: RNA was extracted from fresh PB samples with QIAampRNA blood mini kit (Qiagen, Germany) according to manufacturer's spin protocol.

2. cDNA Synthesis: cDNA synthesis was performed using the Quantitect reverse transcription kit (Qiagen, Germany) according to the manufacturer's protocol.

3. Real-time PCR: Quantification of PTCH1 transcripts was performed by Applied Biosystems Real-Time PCR Software-Step One. Quantitect SYBR Green PCR Kit (QIAGEN, Hilden, Germany) and validated primers of a Quantitect Primer Assay with the primer sets Hs_00427552 and Hs_GAPDH_2_SG (QT01192646) were used 
according to the manufacturer's recommendations (QIAGEN, Hilden, Germany).

4. Amplification was done by denaturation and elongation of chains. PCR system named LightCycler ${ }^{\circledR}$ was used to normalize level of PTCH1 to GAPDH gene expression.

\section{Statistical analysis}

Recorded data were analyzed using the statistical package for the social sciences, version 20.0 (SPSS Inc., Chicago, Illinois, USA). Quantitative data were expressed as mean \pm standard deviation (SD), median, and range. Chi square test $\left(\chi^{2}\right)$ to calculate difference between two or more groups of qualitative variables. ROC curve (Receiver operator characteristic curve): It is a graphic presentation of sensitivity against 1 - specificity. It is done by comparing values of cases to detect a cutoff of certain outcome. Qualitative data were expressed as frequency and percentage. P-value $\leq 0.05$ was considered significant.

\section{RESULTS}

Our cases were segregated into 27 Female and 18 Male; their mean age was $[43.47 \pm 11.65]$ years. Their initial parameters are shown in table 1.

Table (1): Distribution of CML patients in chronic phase according to their all parameters regarding sex, age (years), FISH, PTCH1 expression Level, Initial PCR BCR-BL, 6 months BCR-BL PCR, Hb before treatment, TLC before treatment, PLT before treatment

\begin{tabular}{|l|c|}
\hline \multicolumn{1}{|c|}{ All parameters } & Patients $(\mathbf{n}=\mathbf{4 5})$ \\
\hline Sex & $27(60.0 \%)$ \\
\hline Female & $18(40.0 \%)$ \\
\hline Male & $43.47 \pm 11.65$ \\
\hline Age (years) & $71.76 \pm 16.99$ \\
\hline FISH & $3.05 \pm 2.26$ \\
\hline PTCH1 expression Level & $60.84 \pm 5.54$ \\
\hline Initial PCR BCR-BL & $4.19 \pm 1.14$ \\
\hline 6 months BCR-BL PCR & $9.92 \pm 1.33$ \\
\hline $\begin{array}{l}\text { Hemoglobin (Hb) (g/dL) } \\
\text { before treatment }\end{array}$ & $126.59 \pm 16.7$ \\
\hline $\begin{array}{l}\text { Total leucocytic count (TLC) } \\
\text { before treatment }\end{array}$ & $402.98 \pm 18.92$ \\
\hline $\begin{array}{l}\text { Platelet count (PLT) (mcL) } \\
\text { before treatment }\end{array}$ & \\
\hline
\end{tabular}

On measuring PTCH1 expression level between cases and control groups, there was a statistical significance being higher in cases compared to control group (Table 2).

Table (2): Comparison between patients and control group according to PTCH1 expression level

\begin{tabular}{|c|c|c|c|}
\hline $\begin{array}{c}\text { PTCH1 } \\
\text { expression } \\
\text { Level }\end{array}$ & $\begin{array}{c}\text { Patients } \\
(\mathbf{n}=45)\end{array}$ & $\begin{array}{c}\text { Control } \\
(\mathbf{n}=10)\end{array}$ & $\begin{array}{c}\mathbf{p}- \\
\text { value }\end{array}$ \\
\hline Mean \pm SD & $3.05 \pm 0.26$ & $1.37 \pm 0.71$ & $\begin{array}{c}0.024 \\
*\end{array}$ \\
\hline
\end{tabular}

*: Significant

After 6 months post treatment by first generation TKI (imatinib), 36 patients (80\%) achieved desired molecular response. PTCH1 expression level was significantly higher in patients who did not achieved treatment response than patients with desired molecular treatment response (Table 3 ).

Table (3): Comparison between patients who achieved desired response to treatment and patients who did not achieved desired response at 6 months post treatment according to PTCH1 expression level in patients groups

\begin{tabular}{|c|c|c|c|}
\hline $\begin{array}{c}\text { PTCH1 } \\
\text { expression } \\
\text { Level }\end{array}$ & $\begin{array}{c}\text { Desired } \\
\text { treatment } \\
\text { response } \\
(\mathbf{n = 3 6})\end{array}$ & $\begin{array}{c}\text { No treatment } \\
\text { response } \\
(\mathbf{n}=9)\end{array}$ & $\begin{array}{c}\mathbf{p} \text { - } \\
\text { value }\end{array}$ \\
\hline Mean \pm SD & $2.41 \pm 0.07$ & $3.58 \pm 0.99$ & $0.017^{*}$ \\
\hline
\end{tabular}

Receiver operating characteristics (ROC) curve was used to define the best cut off value of: PTCH1 expression Level, which was $>2$ and for BCR-ABL PCR after 6 months post treatment, it was $>1.6$ (Table 4 and Figure 1).

Accordingly, cases were divided according to PTCH1 expression level into two groups, one with high PTCH1 expression level and the other had low PTCH1 expression level.

Imatinib failure after 6 months of treatment was insignificantly higher in patients with low PTCH1 expression (Figure 2). 
Table (4): Receiver-operating characteristic (ROC) curve for PTCH1 gene expression.

\begin{tabular}{|c|c|c|c|c|c|c|}
\hline Parameters & Cut-off & Sensitivity & Specificity & PPV & NPV & Accuracy \\
\hline PTCH1 expression Level & $>2$ & $88.9 \%$ & $92.2 \%$ & $73.3 \%$ & $97.1 \%$ & $91.0 \%$ \\
\hline $\begin{array}{c}\text { After 6 months PCR-ABL } \\
\text { PCR }\end{array}$ & $>1.6$ & $78.9 \%$ & $75.4 \%$ & $72.1 \%$ & $96.2 \%$ & $82.2 \%$ \\
\hline
\end{tabular}

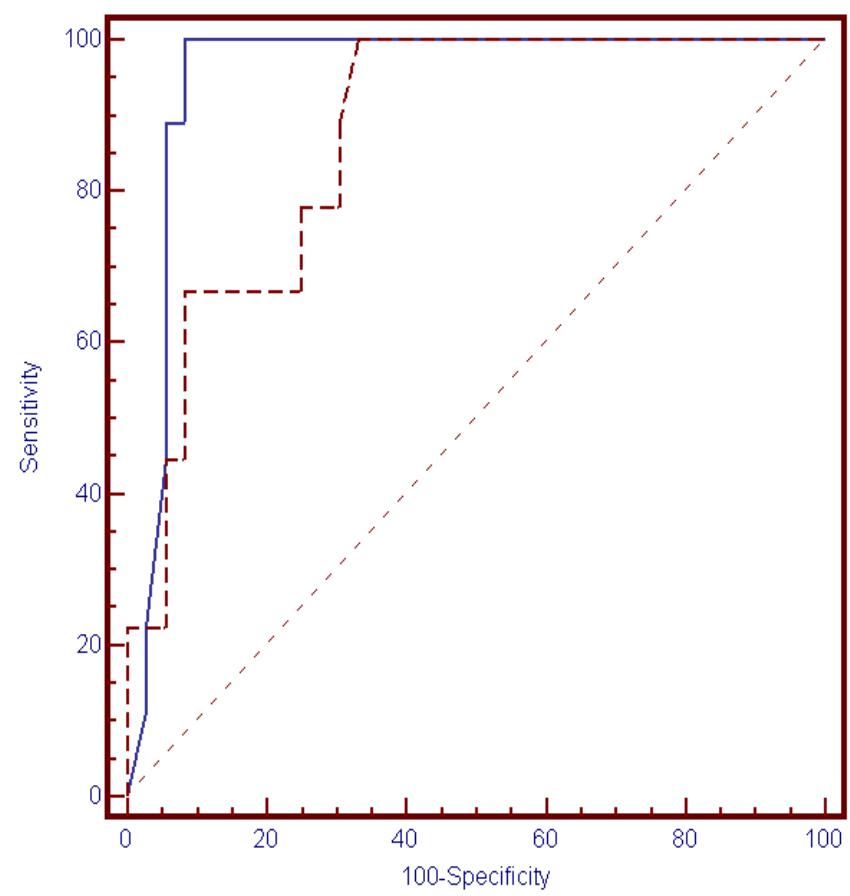

Figure (1): Receiver-operating characteristic (ROC) curve for prediction PTCH1 and PCR for BCR-ABL after 6 months.

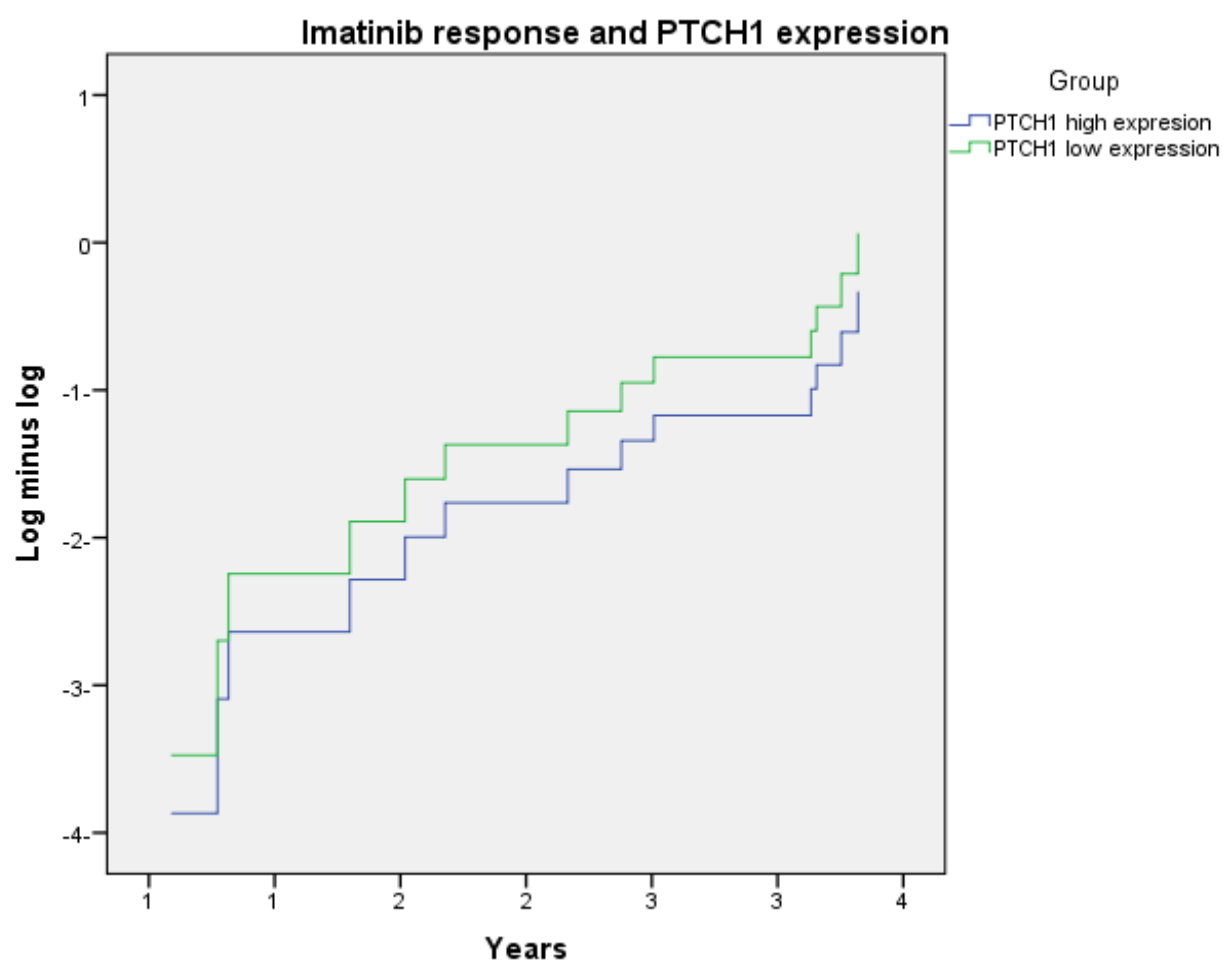

Figure (2): Incidence of imatinib failure after 6 months of treatment compared to low and high PTCH1 expression level. 


\section{DISCUSSION}

The function of the PTCH1 protein is inhibition of the transmembrane protein Smoothened (SMO). Extracellular Hh ligands can attach to the PTCH1 receptor, allowing SMO to signal downstream and activate GLI transcription factors. PTCH1 is classified as a tumour suppressor gene because of its function in inhibiting excessive cell growth. On the other side, SMO functions as an oncogene ${ }^{\left({ }^{(8)} \text {. }\right.}$

In the present study, we evaluated the frequency and prognostic impact of (PTCH1) among a group of Egyptian CML patients. The aim of this study was to use gene expression technology to screen for PTCH1 gene as a possible biomarker and investigate its expression level with imatinib response in CML patients. The final aim was the identification of biomarker as simple prognostic test for TKI response.

In our study, 45 patients were enrolled. Median follow up for patients in our study was 1.9 years. No patient died and there was no instance of transformation to accelerated or blastic crisis phases. The control group consisted of 10 randomly selected age and sex matched healthy subjects.

Juan et al. ${ }^{(9)}$ studied the expression of the PTCH1 gene in chronic-phase CML patients and found that there were no significant differences when comparing the probabilities of CCyR (complete cytogenetic response) between the high and low PTCH1 expression groups, but they found that there were significant differences between the two groups regarding the molecular respone (PCR for BCR-ABL level). The individuals with low PTCH1 expression had a molecular response that was less than ideal. Similarly, we discovered in our study that 44 percent of patients with low PTCH1 expression and 27 percent of patients with high PTCH1 expression developed imatinib resistance after 6 months of therapy, with no statistical significance.

Bing et $\boldsymbol{a l} .{ }^{(10)}$ reported that there were no statistically significant differences of response to imatinib between CML patients with high and low PTCH1 expression and these results were similar to our study. However, more studies on large scale of patients are needed to declare the role of PTCH1 expression in predicting the response of CML patients to first-line TKI treatment, in order to decide whether to start first-line TKI treatment or to choose secondline TKI treatment as an initial line of therapy.

\section{CONCLUSION}

The study demonstrates that level of PTCH 1 did not affect imatinib response. Therefore, PTCH1 may not act as a biomarker for predicting imatinib resistance in CML patients.

Conflict of interest: All authors have no conflict of interest to report.

\section{REFERENCES}

1. Radich J, Deininger M, Abboud C et al. (2019): Chronic Myeloid Leukemia, Version 1.2019, NCCN Clinical Practice Guidelines in Oncology. J Natl Compr Canc Netw., 16(9):1108-1135.

2. Sawyers C, Druker B, Kantarjian H et al. (1999): Activity of an Abl specific tyrosine kinase inhibitor in patients with Bcr-Abl positive acute leukemias, including chronic myelogenous leukemia in blast crisis. In Blood, 94(10): 697A-697A.

3. Jabbour E, Kantarjian H (2018): Chronic myeloid leukemia: 2018 update on diagnosis, therapy and monitoring. Am J Hematol., 93:442-459.

4. Baccarani M, Cortes J, Pane F et al. (2009): Chronic myeloid leukemia: an update of concepts and management recommendations of European Leukemia Net. J Clin Oncol., 27(35):6041-6051.

5. Alonso-Dominguez $\mathrm{J}$, Casado $\mathrm{L}$, Anguita $\mathrm{E}$ et al. (2017): PTCH1 is a reliable marker for predicting imatinib response in chronic myeloid leukemia patients in chronic phase. PLoS One, 12(7): 0181366.

6. Adolphe $\mathrm{C}$, Hetherington $\mathrm{R}$, Ellis $\mathrm{T}$ et al. (2006): Patched1 functions as a gatekeeper by promoting cell cycle progression. Cancer Res., 66(4):2081-8

7. NCCN Guidelines (2021): NCCN clinical practice in oncology guidelines. Chronic Myeloid Leukemia. Version 3. https://www.nccn.org/ guidelines/ category_1

8. Kogerman P, Krause D, Rahnama F et al. (2002): Alternative first exons of PTCH1 are differentially regulated in vivo and may confer different functions to the PTCH1 protein. Oncogene, 21(39):6007-16.

9. Juan M, Dominguez A, García-Delgado $\mathrm{R}$ et al. (2017): Predictive factors for anemia response to erythropoiesis-stimulating agents in myelofibrosis. European Journal of Haematology, 130:5245-59.

10. Bing L, Zhu H, Zhu C et al. (2011): Activation of the Hedgehog pathway in chronic myelogeneous leukemia patients. Journal of Experimental \& Clinical Cancer Research, 30(1):8-13. 